\title{
Cervicofacial necrotizing fasciitis of odontogenic origin: A case report
}

\author{
Manish Tiwari ${ }^{1 *}$, Vikas Meshram ${ }^{2}$, Pravin Lambade ${ }^{3}$ and Gabriela Fernandes ${ }^{4}$ \\ ${ }^{1}$ Park Clinic, 4 Gorky Terrace Rd, Elgin, Kolkata, West Bengal, India \\ ${ }^{2}$ Department of Oral and Maxillofacial Surgery, Government Dental College, Mumbai, India \\ ${ }^{3}$ Department of Oral and Maxillofacial Surgery, SDKS Dental College, Nagpur, India \\ ${ }^{4}$ Department of Oral Biology, School of dental medicine, SUNY Buffalo, New York, USA
}

\begin{abstract}
Necrotizing fasciitis is a progressive, fulminant bacterial infection of the subcutaneous tissue that spreads rapidly through the fascial planes and ultimately leads to extensive tissue destruction. In this paper, we present an extremely rare case of Cervicofacial Necrotizing Fasciitis of Odontogenic origin in a malnourished 60-yearold female of poor socioeconomic conditions, with extensive loss of tissue in the affected region that was managed successfully by functional healing with excellent aesthetic results. Furthermore, we have discussed the keys to early diagnosis and prompt treatment.
\end{abstract}

\section{Introduction}

Cervicofacial necrotizing Fasciitis (NF) is a rare, fatal, rapidly progressive infection of soft tissue that often presents as necrosis of the superficial fascia with undermining of the subcutaneous tissue and necrosis of the skin $[1,2]$. The term necrotizing fasciitis was first coined by Wilson in 1952 [1]. The mortality rate of NF has been reported to be as high as $40 \%$ [3]. Being a rare entity, it is very important for an Oral and Maxillofacial Surgeon to possess knowledge of the cardinal signs and symptoms of this disease and to initiate the treatment as early as possible. Here, we present a case of cervicofacial Necrotizing Fasciitis in a malnourished female of poor socioeconomic conditions caused by Staphylococcus aureus.

\section{Case presentation}

A 60-year-old lady referred to the Oral and Maxillofacial Department, reported with a left-sided extra oral necrotic lesion of the Cervicofacial region with a previous diagnosis of inflammatory swelling. The patient previously noticed a small swelling about a week ago which had grown rapidly to the present size. The patient experienced generalized weakness, bradycardia with complaints of vertigo, malaise, and fever. She also had laborious breathing and immobility of the neck. On examination, the lesion appeared dark, produced an extremely foulsmelling with purulent exudative discharge and poorly defined edges. It was tender on palpation with crepitation and bursting of gas bubbles on compression, soft in consistency and covered by necrotic skin. Extraorally, it extended anteroposteriorly from the midline of the neck to the lateral border of the Trapezius muscle, superoinferiorly it extended from gonial angle till the supraclavicular region (Figure 1). On intraoral examination, it was found that the patient had poor oral hygiene and there were badly carious teeth in the right mandibular quadrant with sublingual edema and pus discharge. The left submandibular lymph node was enlarged, tender on palpation, and freely movable and single in number. The medical history was noncontributory and there was no relevant family history of a similar disease.

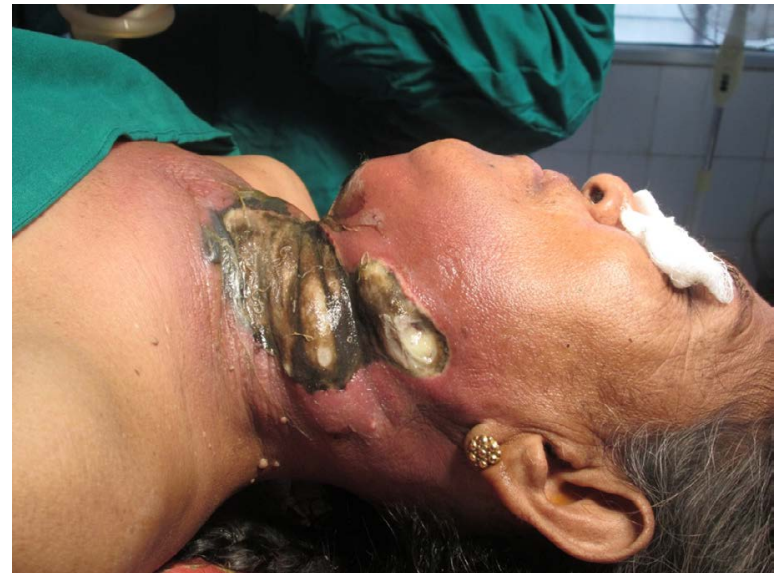

Figure 1. Preoperative Photograph taken at the time of admission, showing the lesion with peculiar greenish black discoloration with necrotic changes, surrounding erythmatous tissue

An orthopantomogram and lateral neck radiograph showed grossly carious second and third molar and an expansile soft tissue lesion of the mandible extending in the neck region. The lesion had multilocular radiolucent areas indicative of presence of gas in the lesion (Figure 2). Routine blood examination showed reduced hemoglobin level (7gm \%) with increased leukocyte count, a swab with the wound exudate was immediately sent for Cultural and Sensitivity Test, so as to identify the predominant species as early as possible. The skin was widely

${ }^{\star}$ Correspondence to: Dr Manish Tiwari, Fellow, Oral Oncology, Park Clinic, 4 Gorky Terrace Rd, Elgin, Kolkata, West Bengal, India, Email: drmanishstiwari@gmail.com

Key words: necrotizing fasciitis, odontogenic infection, cervicofacial, head and neck

Received: June 17, 2018; Accepted: June 22, 2018; Published: June 29, 2018 


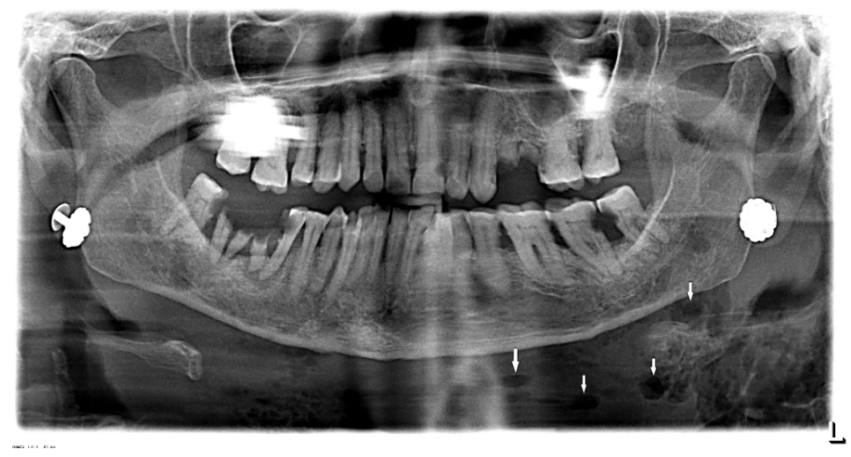

Figure 2. Panoramic Radiograph, showing presence of air pockets at the site of the lesion

undermined by the progressing infection and it was dusky to black and showed necrotic changes with burst of air bubbles on slight finger pressure. The lesion was extremely foul smelling. Correlating with the age, progress, clinical behavior \& radiological pattern, the lesion was diagnosed as Cervicofacial Necrotizing Fasciitis of Odontogenic Origin.

The patient was immediately administered with intravenous fluids and a combination of Cefuroxime, Gentamicin and Metronidazole antibiotics empirically with analgesic and anti-inflammatory drugs. The lesion was covered with a sterile dressing. On the same day, two points of blood transfusion were done as the patient was anemic and the surgery was planned under general anesthesia. Patient was draped and prepared under all aseptic precautions; Clinical inspection of the wound demonstrated that the subcutaneous fat has no structural integrity and offers little resistance to the exploring finger.

Through transverse neck incision the tissue planes over the superficial layer of deep cervical fascia was explored and all the lacunae filled with pus were explored until normal tissues were found. After surgical exploration using hemostat and blunt finger dissection, very foul smelling gray color pus with dead tissues were obtained with frequent air bubbles in it. Extensions of the lesion was found in the submental, supraclavicular spaces, they were also explored and debrided. The grossly carious teeth were extracted. Copious irrigation was done with hydrogen peroxide enhanced betadine solution and Metronidazole. The wound was covered with magnesium sulfate supra added dressing. A second debridement was performed after two days.

In this case, Cefuroxime was found to be resistance to the pathogenic organism in sensitivity test, and it was replaced with a combination of Amoxicillin and Clavulanic acid which was found to be effective in the cultural sensitivity test. The chief pathogenic organism found was Staphylococcus aureus along with anaerobic flora.

On a daily basis, aggressive dressing was done using Povidone Iodine with Hydrogen peroxide irrigation, Chloromycetin ointment application. On the seventh-day healthy granulation tissue and the vascular bed was observed (Figure 3a) on the muscles and deep structures of the neck since then a less frequent dressing was done. The patient was shifted on oral medications and kept on a diet rich in protein, vitamin A and C. functional physiotherapy of the patient was started for functional healing. On 45th day, the submental defect was completely healed and the defect in neck region had sloping edges with new healthy epithelium on the wound bed. After 3 months satisfactory healing was noticed with minimal contraction and without any loss of function (Figure $3 b$ ).

\section{Discussion}

NF is defined as a relatively rare infection; it is characterized by rapidly progressing necrosis of the fascia and subcutaneous fat, with subsequent necrosis of overlying skin. In the Head and neck region it usually occurs secondary to immunocompromised state of host, development of NF is enhanced by Otologic infections, cervical adenitis, Peritonsillar abscess, Odontogenic infections [4] and Salivary gland infections. Approx. $60 \%$ of cases are polymicrobial, including one or more obligate anaerobes (Synergistic Gangrene).

The most critical early distinctive feature of NF is a disproportionate pain in comparison with physical findings. On physical examination even, a wound which seems small may lead to severe pain. Rapidly developing inflammatory skin lesions including heat and erythema can be seen as the early clinical features [5]. Thrombosis of nutrient vessels supplying the skin leads to skin necrosis. Patient loses perception due to damage to underlying nerves and ultimately there is discharge of purulent necrotic substance on the surface (Figure 4) [3].

The patients present with blister and bullae formation in late phages of disease with extravasations of purulent discharge. In severe cases Patient may show symptoms of septic shock, such patients carry very high chance of mortality thus resuscitation should be started as early as possible. The disease must be recognized very quickly. Paucity of specific cutaneous signs makes early recognition extremely difficult, thus diagnosis of NF depends on the rapid progress of disease and inability of patient to respond to antimicrobial therapy.

The final diagnosis is confirmed by surgical exploration which reveals grey dead tissues in the subcutaneous space. Immediate broadspectrum antibiotics which can be modified once culture results are obtained and surgical debridement of the lesion are the key to good prognosis [6]. At our institute, agents used to reduce microbial load include diluted Povidone-iodine, hydrogen peroxide $\left(\mathrm{H}_{2} \mathrm{O}_{2}\right)$, and
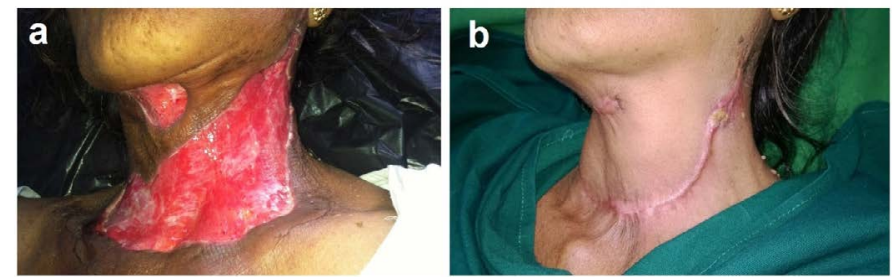

Figure 3(a). Seventh day post-operative Photograph, showing the formation of healthy granulation tissue and vascular bed (b) 3rd month post-operative Photograph showing esthetically acceptable healing of the defect without much wound contraction, restoring the normal function

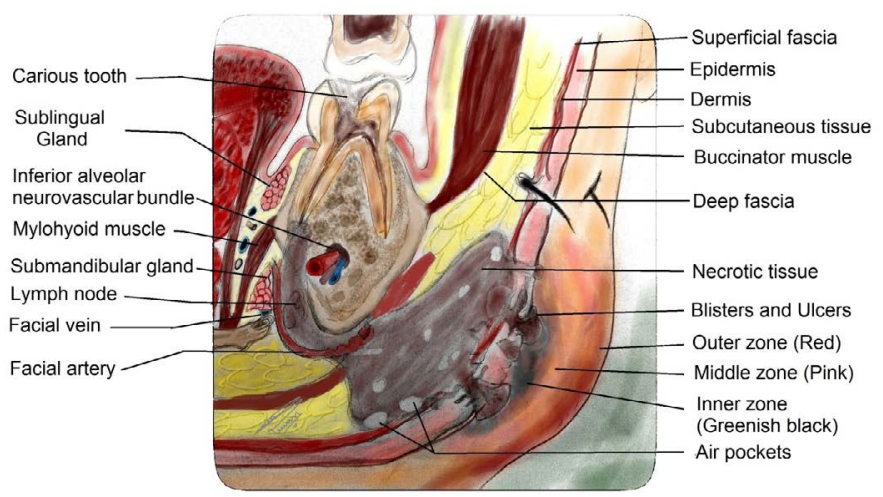

Figure 4. Schematic diagram showing Spread of Necrotizing Fasciitis of Odontogenic origin 
Metronidazole. In the longer term, wounds dressing was done with magnesium-sulfate supra-added dressing. Hyperbaric oxygen therapy (one to three dives) has been suggested as a supportive measure, however it may not be a feasible alternative because of economic reasons. Further surgical management for the coverage of the wound or secondary healing can be considered once the patient is stable; once the wound bed demonstrates healthy granulation tissue [7].

In our patient, by examining the size and the extent of the lesion, graft placement for the coverage of wound would have been the first choice. But because the risk of graft rejection is elevated in infected wounds, the patient's unwillingness for second surgery and to avoid donor site morbidity use of graft was inadvisable in this case. We started functional physiotherapy of the patient and proper wound care by doing dressing at regular intervals using Povidone iodine and Chloromycetin ointment to decrease the bacterial load. The unhealthy margins were removed during each exposure for the wound dressing; the wound bed was kept moist to allow for easier epidermal migration and neoangiogenesis and thereby achieving rapid healing. There are evidences which show that moist wound healing results in better cosmesis, decrease pain, and improvement in the granulation tissue of wound bed [8]. With meticulous wound care and multiple attempts made to reverse the patient's Immunocompromized state a satisfactory healing was achieved without need of graft and also without much wound contraction or loss of function.

In India, poor socioeconomic status, malnutrition, ignorance of primary dental infections, delay in referral and long distance to the tertiary hospitals are the main problems patient have to face with. India accounts for the largest number of anemic patients in the world [9], the anemia have major contribution to the rapid spread of infection and it is considered as the cause of making patients more vulnerable for the onset of Odontogenic infections. Thus all required steps should be taken to reverse these systemic morbidities along with the surgical and supportive care measures.

\section{Conclusion}

The Cervicofacial necrotizing Fasciitis can always be a cause of death if not intervened promptly; this extensive disease entity is commonly caused by an Immunocompromized state of host, malnutrition, diabetes, and ignored dental infections, surgical and non-surgical trauma. The mainstay of the treatment includes prompt surgical debridement, intravenous antibiotics, fluids and electrolytes management and analgesia along with meticulous attempt to reverse the immunocompromized state of the patient, thus improving their general condition. This case report presents a successfully treated case of Cervicofacial necrotizing fasciitis secondary to Odontogenic infection with extensive loss of tissue in the affected region that has been managed by functional healing via secondary epithelisation in order to produce satisfactory esthetic results.

\section{Conflict of interest}

None.

\section{Patient consent}

The patient gave her informed consent prior to her inclusion in the study. Details that might disclose the identity of the patient were omitted.

\section{Ethical standards}

For this type of study formal consent is not required.

\section{References}

1. Miller JD (1983) The importance of early diagnosis and surgical treatment of necrotizing fasciitis. Surg Gynecol Obstet 157: 197-200. [Crossref]

2. Martínez AY, McHenry CR, Meneses Rivadeneira L (2014) Fasceítis necrosante cervicofacial: una infección severa que requiere tratamiento quirúrgico temprano. Rev Esp Cir Oral Maxilofac.

3. Özkan, A., Sentürk, S., Topkara, A, Tosun Z (2015) Extensive cervicofacial necrotizing fasciitis of odontogenic origin: case report and literature review. Eur J Plast Surg 38: 143.

4. McGurk M (2003) Diagnosis and treatment of necrotizing fasciitis in the head and neck region. Oral Maxillofac Surg Clin North Am 15: 59-67. [Crossref]

5. Melaney F Hemolytic Streptococcus gangrene. Arch Surg 9317 - 64:1924

6. Ali MH, Zayed ME (1997) Necrotizing fasciitis of the head and neck: Report of three cases. Ann Saudi Med 17: 641-645. [Crossref]

7. KC Toran, Nath S, Shrestha S, Rana BBS JB (2004) Odontogenic origin of necrotizing fasciitis of head and neck - a case report, Kathmandu University Medical Journal. 2;361-3:2004

8. Helfman T, Ovington L, Falanga V (1994) Occlusive dressings and wound healing. Clin Dermatol 12: 121-127. [Crossref]

9. Gragnolati M, Shekar M, Gupta MD, Bredenkamp C, yi- Kyoung Lee (2005) India's undernourished children: a call for reform and action.HNP publications-the World Bank.

Copyright: (C2018 Tiwari M. This is an open-access article distributed under the terms of the Creative Commons Attribution License, which permits unrestricted use, distribution, and reproduction in any medium, provided the original author and source are credited. 